\title{
Measurement Science is the Science of Sciences - There is no Science without Measurement
}

\author{
Viktor Witkovský, Ivan Frollo \\ Institute of Measurement Science, Slovak Academy of Sciences, Dúbravská cesta 9, 84104 BRATISLAVA, Slovak Republic, \\ witkovsky@savba.sk
}

\begin{abstract}
Omnia in mensura et numero et pondere disposuisti is a famous Latin phrase from Solomon's Book of Wisdom, dated to the mid first century $\mathrm{BC}$, meaning that all things were ordered in measure, number, and weight. Naturally, the wisdom is appearing in its relation to man. The Wisdom of Solomon is understood as the perfection of knowledge of the righteous as a gift from God showing itself in action. Consequently, a natural and obvious conjecture is that measurement science is the science of sciences. In fact, it is a basis of all experimental and theoretical research activities. Each measuring process assumes an object of measurement. Some science disciplines, such as quantum physics, are still incomprehensible despite complex mathematical interpretations. No phenomenon is a real phenomenon unless it is observable in space and time, that is, unless it is a subject to measurement. The science of measurement is an indispensable ingredient in all scientific fields. Mathematical foundations and interpretation of the measurement science were accepted and further developed in most of the scientific fields, including physics, cosmology, geology, environment, quantum mechanics, statistics, and metrology. In this year, 2020, Measurement Science Review celebrates its 20th anniversary and we are using this special opportunity to highlight the importance of measurement science and to express our faith that the journal will continue to be an excellent place for exchanging bright ideas in the field of measurement science. As an illustration and motivation for usage and further development of mathematical methods in measurement science, we briefly present the simple least squares method, frequently used for measurement evaluation, and its possible modification. The modified least squares estimation method was applied and experimentally tested for magnetic field homogeneity adjustment.
\end{abstract}

Keywords: Measurement science, linguistic interpretation, measuring process, physical phenomena, measurement evaluation, magnetic field.

\section{INTRODUCTION}

Each measuring process assumes an object of measurement. However, each object must be localized in space; in general, there can be no space without objects.

Objects can be visible, palpable but also without visual characteristics, e.g. electricity, magnetism. Everything we perceive as reality is a process that appeals to our consciousness and only then can be investigated by way of measurement. It should be accepted that physical phenomena existed and exist before they are measured. Humanly understandable explanation of how the world looks like comments the so called biocentrism [1].

Some science disciplines, such as quantum physics, are still incomprehensible despite complex mathematical interpretations. The time factor, as we know it, challenges quantum theory. It must therefore be noted that no phenomenon is a real phenomenon unless it is in a state of observation in space and time and be subject to measurement. And therefore, the science of measurement is a science in all fields of sciences. Measurement without affecting the measured object, as accurately as possible, is the main attribute of the measurement science.
The idea that there is no science without measurement was first proclaimed by William Thomson - who was also wellknown as Lord Kelvin, an Irish-Scottish mathematical physicist and engineer born in Belfast in 1824. At the University of Glasgow, he did an important work in mathematical analysis of electricity [2]. He declared that "When you can measure what you are speaking about, and express it in numbers, you know something about it, when you cannot express it in numbers, your knowledge is of a meager and unsatisfactory kind; it may be the beginning of knowledge, but you have scarcely, in your thoughts advanced to the stage of science." His interpretation of the measurement science, in the sense of the mathematical foundations, was accepted and further developed by his followers, e.g. [3]-[8], in cosmology, geology, environment, quantum mechanics, statistics, and metrology.

In a similar vein, Ishikawa emphasizes in [9]: "I proposed measurement theory, which is characterized as the metaphysical and linguistic interpretation of quantum mechanics. I assert that this theory is one of the most fundamental languages in science, and thus, it is located at the central position in science." 
Even today, the claim that measurement science is the science of sciences and there is no science without measurement is convincingly confirmed. On the other hand, measurement science is a mature, independent and unique scientific discipline represented by many respected scientific journals publishing the results from various areas of basic and applied research aimed at developing measurement methods and analyzing measurement data. Of these, ten scientific journals registered in the Web of Sciences Base Collection database contain the term "Measurement" in their title, and two of them include the explicit expression "Measurement Science".

Similarly, many research institutions, companies, departments and universities around the world devote themselves to the development of measurement sciences and use the term ,measurement” in their name.

We are glad and proud that the Institute of Measurement Science of the Slovak Academy of Sciences (IMS SAS) is a part of this important category of academic institutions which contributes significantly to the development of knowledge in the field of measurement science. Its activities encompass fundamental and applied research. In particular, measurement theory and mathematical-statistical methods for processing of experimental results, systems for measuring selected physical quantities, development of measuring systems, models, and processing methods for bio signals, and other non-standard problems in applied sciences and industry. In addition, IMS SAS is the organizer of regular bi-annual conferences MEASUREMENT devoted to different aspects of the measurement science, and founder and sole owner of the scientific journal Measurement Science Review (MSR), which celebrates its $20^{\text {th }}$ anniversary, as it has been successfully published since September 2001 under supervision of its founding Editor-in-Chief, Professor Ivan Frollo.

The aim of the journal is to publish papers from scientific disciplines covering the measurement science with an orientation toward the theory of measurement, measurement of physical quantities and measurement in biomedicine. We believe that the MSR is providing an environment for information exchange among scientists, engineers and industry people, as well as encouragement of effective and coordinated research cooperation. The advantage of the journal is the speed of publication, high reputation, and open access policy for all published papers.

We use this exceptional opportunity to express our belief that the journal MEASUREMENT SCIENCE REVIEW will continue to be an excellent place for exchanging bright ideas, experiences and good practices, establishing new contacts for common projects, and making new friends in the field of measurement science.

\section{SUBJECT \& METHODS}

A frequent method for analysis of measurement results, based on using the statistical regression modeling approach, is the least-squares approach, used to approximate the solution of overdetermined systems by minimizing the sum of the squares of the residuals made in the results of every single equation.
However, when the problem has substantial uncertainties in the independent variable, then simple regression and leastsquares methods may have problems and the methodology required for fitting the errors-in-variables models may be considered instead of the standard regression model with the simple least squares estimation approach. A further complication arises when additional restrictions on the model parameters should be applied.

As an illustration and possible motivation for usage and further development of mathematical methods in measurement science, in this note we present only the simple least squares method, frequently used for measurement evaluation, and consider the need of its possible modification caused by the imposed restrictions on the parameters.

We start with an example of examination of the possibilities of multi-parameter measurements on the selected object with options:

- without affecting the parameters of the object,

- affecting the parameters of the object with the possibility to set the selected parameters according to predefined objectives.

Pre-defined selected parameters of the object may be oriented to phenomena in the field of electrical and magnetic fields, optical phenomena, temperature phenomena, i.e. phenomena, which are presented in the form of a physical field.

In general, these measurements, when properly implemented with high accuracy, are acceptable and provide a clear set of data that can be used to correct the parameters of the measured object into the state we require and which is physically accomplishable, [10].

Obviously, the methodology for correcting parameters has an infinite number of solutions. The aim is to find an optimal set of corrections that could be reasonably feasible, [11].

\section{A. Least squares method for measurement evaluation}

Here we consider the task of estimation of the unknown parameters of a linear regression function as used and applied in [11]. It is a problem where measurements of real values are performed. We shall assume that the theoretical expected values of the measured values, say $r_{1}, r_{2}, \ldots, r_{n}$, are expressed as a linear combination of unknown parameters $K_{1}, K_{2}, \ldots, K_{p}$, with $p \leq n$.Then the determining equation for our task is

$$
E\left(r_{i}\right)=v_{1 i} K_{1}+v_{2 i} K_{2}+\cdots+v_{p i} K_{p}
$$

for $i=l, 2, \ldots, n$, where $v_{1 i}, v_{2 i}, \ldots v_{p i}$ are known values of measuring object differences corresponding to known testing results.

Let $\mathbf{M}=\left\|v_{j i}\right\|$ be a $p \times n$ matrix of coefficients for $i=l, 2, \ldots$, $n, j=l, 2, \ldots, p$, where the known coefficients $v_{1 i}, v_{2 i}, \ldots, v_{p i}$ from equation (1) are stacked into the i-th column of the matrix M. Moreover, we shall suppose that the rows of the matrix $\mathbf{M}$ are linearly independent.

Based on the standard least squares theory, the unknown values of the parameters $K_{1}, K_{2}, \ldots, K_{p}$ are estimated by minimizing the sum of squared residuals with respect to the possible values of the unknown parameters, specified by 


$$
\mathbf{S}=\sum_{i=1}^{n}\left(r_{i}-v_{1 i} K_{1}-v_{2 i} K_{2}-\cdots v_{p i} K_{p}\right)^{2}
$$

Then the least-squares estimates, here denoted as $K_{1}^{*}, K_{2}^{*}, \ldots, K_{p}^{*}$, are determined by solving the system of normal equations,

Let

$$
\frac{\partial S}{\partial K_{h}}=0 \text { for } \mathrm{h}=1,2, \ldots, p .
$$

$$
\begin{gathered}
b_{h j}=\sum_{i=1}^{n} v_{h i} v_{j i} \quad(h, j,=1,2, \ldots p) \\
b_{h}=\sum_{i=1}^{n} v_{h i} r_{i} \quad(h=1,2, \ldots p),
\end{gathered}
$$

And let us denote $\mathbf{B}=\left\|b_{h j}\right\|$, and $\mathbf{b}=\left\|b_{h}\right\|$. In fact, by using the matrix notation we get

$$
\begin{aligned}
\mathbf{B} & =\mathbf{M M}^{\prime} \\
\mathbf{b} & =\mathbf{M r}
\end{aligned}
$$

where $\boldsymbol{r}=\left(r_{1}, r_{2}, \ldots, r_{n}\right)^{\prime}$ is a vector of the observed values of the object, $\mathbf{M}^{\prime}$ is a transpose matrix to the matrix $\mathbf{M}$, matrix $\mathbf{B}$ is a symmetrical matrix of size "p x p" depending on known coefficients $v_{j i}$ of the determining equation (1).

The normal equations are now expressible in the form

$$
\mathbf{B} \boldsymbol{K}^{*}=\mathbf{b}
$$

Then, the least-squares estimate $\boldsymbol{K}^{*}=\left(K_{1}^{*}, K_{2}^{*}, \ldots, K_{p}^{*}\right)^{\prime}$ of the vector parameter $\boldsymbol{K}=\left(K_{1}, K_{2}, \ldots, K_{p}\right)^{\prime}$ is given by

$$
\boldsymbol{K}^{*}=\boldsymbol{B}^{-1} \mathbf{b}
$$

Using equation (6) and equation (7) we get the solution of equation (3) in a matrix form:

$$
\mathbf{K}^{*}=\left[\mathbf{M} \cdot \mathbf{M}^{\prime}\right]^{-1} \cdot \mathbf{M} \cdot \mathbf{r},
$$

where $\left[\mathbf{M} . \mathbf{M}^{\prime}\right]^{-1}$ is an inversion matrix to the matrix $\left[\mathbf{M} . \mathbf{M}^{\prime}\right]$, and $\mathbf{K}^{*}=\left(K_{1}^{*}, K_{2}^{*}, \ldots, K_{p}^{*}\right)^{\prime}$ is the estimated vector of the contributions. Simple solution of our problem is given by the following equation:

$$
\mathbf{M}^{\prime} \mathbf{K}^{*}=\boldsymbol{r}^{*}
$$

where $\boldsymbol{r}^{*}$ is the vector of fitted values and $\boldsymbol{r}-\boldsymbol{r}^{*}$ is the vector of residuals such that the sum of squared residuals is minimum.

It is necessary to remark that the vector of estimated parameters $\mathbf{K}^{*}$, the vector of fitted values $\boldsymbol{r}^{*}$, and the vector of residuals, $\boldsymbol{e}=\boldsymbol{r}-\boldsymbol{r}^{*}$, are practical values related to the physical object after homogeneous components subtraction.

By using the contribution energy, we are able to correct only the differences of the physical object values from its mean value in a selected interval. Computing of the contribution values according to equation (10) or equation (11) is exact. But in practice this method does not necessarily lead to a reasonable solution because the estimated contribution values can be higher than, e.g. the power supply possibilities.

\section{B. Practical computing algorithm}

In general, the least squares algorithm is easy to implement and nowadays it is available in most software packages suggested for statistical and/or technical computing.

In [11] we have considered the problem of magnetic field correction of a home-made whole-body NMR imager 0.1 Tesla. We were looking for such correcting values that minimize the physical object inhomogeneity, respecting technical parameters of the equipment. For that we have suggested to use an algorithm based on the least squares method. It was necessary to find a criterion for the final quality of the physical object parameters. In the described method we have used a minimization procedure of the mean square deviation of the physical object parameters in selected points. The presented algorithm calculating with limited values in a real range was implemented by using the software system Mathematica (Wolfram Research). The basic sequence of the solution is evident from Fig.1. The algorithm is using suitable built-in functions, like e.g. FindMinimum(S), where $\mathrm{S}$ is specified by equation (2).

For our task we can use the following sequence:

1. Measurement of the magnetic field $\mathbf{r}_{\mathrm{i}}$ in all points of selected planes, join data.

2. Mean value calculation: $\sum \frac{r_{i}}{n}=r_{m}$, oscillating component: $r_{b}=r_{i}-r_{m}$.

3. Primary inhomogeneities estimation: $r_{\text {inhom }}=\operatorname{Max}\left[r_{i}\right]-\operatorname{Min}\left[r_{i}\right]$.

4. Measurement of physical object parameters contributions of every contribution in all selected points: $\left[v_{1 i}, v_{2 i}, \ldots v_{p i}\right]$.

5. Basic equation construction: equation (2).

6. Find minimum of the target function $\mathrm{S}$.

7. Output values: $K_{1}^{*}, K_{2}^{*}, \ldots, K_{p}^{*}$.

8. Test using equation (2).

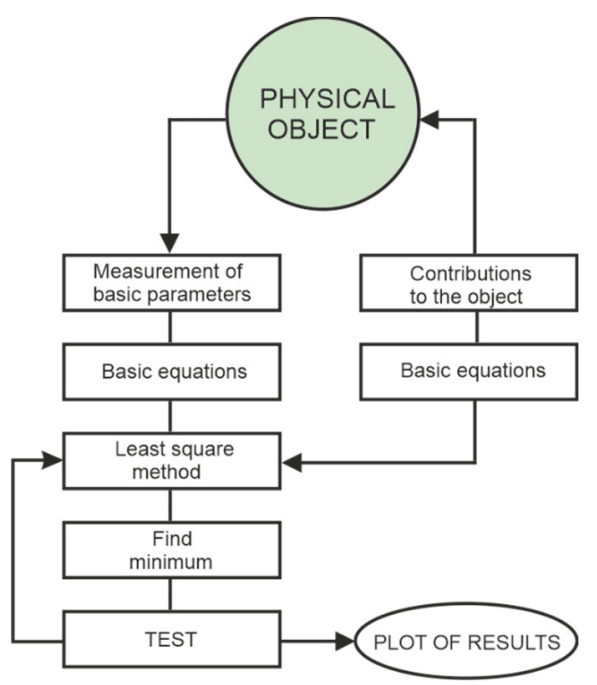

Fig.1. Flowchart of the main measuring and optimizing procedures. 
The calculated values were verified by adjustment of the contributions for every point of the physical object and by the experimental measurements of the resultant physical object parameters.

\section{RESULtS}

We have demonstrated a least squares method for optimization of the basic parameters for selected physical experiment design where large input parameter adjustment is needed. We have shown how to use this method for stationary magnetic field homogeneity calculation. The homogeneous magnetic field is a basic condition for imaging based on nuclear magnetic resonance. The designed method can be used for regular testing of a basic electromagnet and for shim coil currents adjusting with the goal to create optimal conditions of NMR experiments. The speed of calculation and experimentally verified results are promising to use this method by many physical projects where large input parameter adjustment is needed.

It is necessary to say that the demonstrated example is only a very limited method for affecting the parameters of the object with the possibility to set the selected parameters according to predefined objectives.

Interesting example oriented to static magnetic field was published in [12]. Spatial distribution of magnetic field is determined by vector shape of the Laplace differential equation. Magnetic field is expressed in the form of the development of spatial spherical functions transferring to the rectangular coordinate system, [13]. The individual values of the operative parameters on the surface are found by measurement. The sum of the squared differences in particular points of measurement belonging to the bounding area on the surface of the sphere was calculated. To minimize error on the measurement area, the least squares method was used. The final numerical calculation submitted the values for correcting coils currents adjustment and magnetic field of the whole-body electromagnet for MRI with highest homogeneity adjusted.

\section{DISCUSSION}

Measurement science is the basis of all experimental and theoretical research activities. The Institute of Measurement Science as a representative of an academic institution focused on the measurement science is publishing the journal Measurement Science Review. MSR now celebrates its 20th anniversary under supervision of its founding Editor-inChief, Professor Ivan Frollo. Here we took this opportunity to highlight the importance of measurement science and our belief that the journal will continue to be an excellent place for exchanging bright ideas, experiences and good practices, establishing new contacts for common projects, and making new friends in the field of measurement science.

As an illustration and motivation for usage and further development of mathematical methods in measurement science, we have presented the simple least squares method, frequently used for measurement evaluation, and its possible generalization, as used in the errors-in-variable model for comparative calibration.

\section{ACKNOWLEDGMENT}

The support by the Slovak Research and Development Agency (projects APVV-15-0029 and APVV-15-0295) and the Scientific Grant Agency of the Ministry of Education of the Slovak Republic and the Slovak Academy of Sciences (projects VEGA 2/0054/18, VEGA 2/0081/19, and VEGA $2 / 0003 / 20$ ) is greatly acknowledged.

\section{REFERENCES}

[1] Berman, B., Lanza, R. (2010). Biocentrism: How Life and Consciousness are the Keys to Understanding the True Nature of the Universe. BenBella Books.

[2] Lord Kelvin`s Quotations: https://zapatopi.net/kelvin/quotes/\#meas, https://www.quotetab.com/quotes/by-lord-kelvin

[3] Emiliani, C. (2007). Planet Earth: Cosmology, Geology, and the Evolution of Life and Environment. Cambridge Univesity Press.

[4] Di Giuseppe, A. (2013). Metrology and Physical Constants. IOS Press.

[5] Seddon, D. (ed.) (1978). Relations of Production: Marxist Approaches to Economic Anthropology. London: Frank Cass.

[6] Ishikawa, S. (2006). Mathematical Foundations of Measurement Theory. Keio University Press Inc.

[7] Ishikawa, S. (2012). Quantum mechanics and the philosophy of language: Reconsideration of traditional philosophies. Journal of Quantum Information Science, vol. 2 , no. 1, p. 2-9.

[8] Clark, M. (2007). Statistics: A clarification. University Information Technology.

[9] Ishikawa, S. (2012). Measurement theory in the philosophy of science. arXiv:1209.3483 [physics.histph].

[10] Yamamoto, S., Yamada, T., Morita, M., Matsuda, T., Iwamoto, M. (1985). Field correction of a highhomogeneous field superconducting magnet using a least squares method. IEEE Transactions on Magnetics, vol. 21, no. 2, p. 698-701.

[11] Frollo, I., Andris, P., Strolka I., Bačiak, L. (2005). A least square method for measurement and optimisation in selected physical experiments. In Measurement Technology and Intelligent Instruments VI. UetikonZuerich, Switzerland: Trans Tech Publications Ltd., p. 681-686. ISBN 0-87849-977-6.

[12] Frollo, I., Andris, P., Krafčík, A., Gogola, D., Dermek, T. (2018). Magnetic field homogeneity adjustment for magnetic resonance imaging equipment. IEEE Transactions on Magnetics, vol. 54, no. 5, p. 6500309.

[13] Chen, H.-H., Boykin, R.D., Clarke, G.D., Gao, J.-H.T., Roby, J.W. (2006). Routine testing of magnetic field homogeneity on clinical MRI systems. Medical Physics, vol. 33, no. 11, p. 4299-4306. 
Doc. RNDr. Viktor Witkovský, CSc., is a senior researcher at the Institute of Measurement Science, Slovak Academy of Sciences in Bratislava, Slovakia. In 1986 Viktor graduated from the Faculty of Mathematics and Physics of Comenius University in Bratislava (now Faculty of Mathematics, Physics and Informatics), specialization Probability and Mathematical Statistics. He received his $\mathrm{PhD}$ at the Mathematical Institute of the Slovak Academy of Sciences in 1993. From 1998 to 2018 he worked there as the head of the Department of Theoretical Methods, from 2006 to 2018 he was Deputy Director of the Institute and currently (from 2019) he works as the director of the IMS SAS. His scientific focus is on the development of mathematical-statistical models for measurement processes, as well as the development of methods and algorithms for the analysis and expression of uncertainties in measurement and metrology. He is the author or co-author of 48 articles published in scientific journals registered in the Web of Science database, three domestic monographs and more than 60 conference papers, presentations and other publications with more than 500 citations.

His international recognition is documented by a number of invited lectures, membership in the editorial boards of international scientific journals, membership in international organizations, participation in the development of international ISO standards, as well as serving as regular reviewer of scientific project proposals and manuscripts submitted for publication in scientific journals.

Prof. Ing. Ivan Frollo, DrSc., is a senior researcher and head of the Department of Imaging Methods at the Institute of Measurement Science of the Slovak Academy of Sciences in Bratislava, Slovakia. He is an internationally recognized expert in the field of Measurement Technology. He achieved significant results in areas such as measurement methods and instruments for biology, physiology, cardiac surgery and respirology, microcomputer-based electronic measuring instruments for physiology and tomographic methods and nuclear magnetic resonance systems with applications for physics and medicine.

He has long been engaged in magnetic resonance imaging methods helium lung imaging, physics and hyperpolarization physics and methodology, and digital radiological systems. Through his scientific and research activities, he has a long-term impact on the development of measurement technology, especially with focus on measurements in medical sciences.

Ivan Frollo comes from the city of Prešov in eastern Slovakia. In 1963 he graduated from the Faculty of Electrical Engineering of the Slovak Technical University in Bratislava (Ing.), specializing in radioelectronics. Between 1963 and 1966 he completed his internal aspiration at the Institute of Measurement Theory of SAS. Since 1966 he has been working at the Slovak Academy of Sciences, first as an independent researcher and since 1985 as a senior researcher. From 1998 to 2006 he worked as the director of the Institute of Measurement Science SAS. At present, he is the head of the Department of Imaging Methods of the IMS SAS.
From 1977 to 1978, he completed a six-month study stay at the University of California, Berkeley, the Department of Electrical Engineering and Computer Science, and 15 other US universities. He completed a number of short-term stays at research centers in Great Britain, Germany, Italy, France, Sweden, Finland, Ireland, Poland, Russia and the USA. He has participated and presented his lectures at many international scientific conferences.

In 1992 he defended his doctoral dissertation (DrSc.) focused on the Measurement Methods and Systems for Medical Research. In 1993 he was habilitated as associate professor and in 1995 he was appointed a university professor in the field of Measurement Technology at the Faculty of Mechanical Engineering of the Slovak Technical University (FME STU) in Bratislava. He also worked for a long time at the Faculty of Electrical Engineering and Computer Science of STU (FEI STU). In 1994-2004 he lectured and led exercises for foreign students, subject: Medical Imaging Techniques, Principles and Instrumentation, to which he published textbooks (1995). His further cooperation with the FME STU and FEI STU consists in supervising the student's diploma theses, he is a member of the committees for state final examinations and for the defense of doctoral dissertations.

During his career he has achieved significant scientific results. He designed and implemented with his team 41 devices and devices that have found application in research and practice. Many of the results of his scientific work have been published and patented.

$\mathrm{He}$ is a member of the Scientific Board for Electronics and Material Technology of the Slovak Academy of Sciences and was chairman of the Commission for PhD study: Bionics and Biomechanics. In 1998 - 2006 he was held the position of director of the Institute of Measurement Science. $\mathrm{He}$ is a member of the Slovak Cybernetic Society, member of the Slovak Medical Society and member of The Engineering in Medicine and Biology Society of the IEEE. Since 1997 he is a member and representative in IMEKO TC7 (Technical Committee on Measurement Science) for the Slovak Republic. Since 2001 until now he is Editor-in-Chief of the journal Measurement Science Review, ISSN 1335 - 8871.

He has been awarded several major awards, as e.g. Silver and Gold Andrej Stodola Plaque of SAS "For Merit in Technical Sciences" (1989 and 1999), Gold Medal of Johann Andrea von Segner " on the 85th Anniversary of Metrology in Slovakia" (2004), SAS Medal for Support of Science (2005), Great Medal of St. Gorazd for scientific and educational activities awarded by the Ministry of Education (2005), Gold Medal of SAS for "Lifelong Work in the Field of Science" (2009), SAS Award "For Popularization of Science" (2015).

In 2019, Professor Ivan Frollo was awarded the Science and Technology Award for his Lifetime Achievement in Science and Technology, in particular for his achievement in the field of Measurement Technology, by the Ministry of Education, Science, Research and Sport of the Slovak Republic.

Received November 13, 2019

Accepted January 30, 2020 